\title{
THE MILLER PROCEDURE FOR MOBILE FLAT FEET
}

\author{
R. K. FRASER, M. B. MENELAUS, P. F. WILliams, W. G. COLE
}

From the Royal Children's Hospital, Melbourne, Australia

\begin{abstract}
We studied the long-term results of the Miller operation at a mean age of 13 years in 22 patients ( 38 feet) with persistently symptomatic mobile flat feet associated with an isolated naviculocuneiform break.

At a mean of 12 years ( 3 to 27 ) after surgery, $84 \%$ of the feet had a satisfactory clinical result. We conclude that the Miller operation is a useful procedure for adolescent patients with persistently symptomatic flat feet with an isolated break at the naviculocuneiform joint.
\end{abstract}

J Bone Joint Surg [Br] 1995:77-B:396-9.

Received 23 August 1994; Accepted 5 October 1994

In adolescent patients, mobile flat feet are usually asymptomatic; minor complaints of foot strain and excessive shoe wear can be relieved by the use of orthoses (Coleman 1983). A few patients, however, may have severe persisting symptoms. The medial longitudinal arch of mobile flat feet with a break at the naviculocuneiform joint can be restored by full dorsiflexion of the great toe as can feet with a mild break at the talonavicular joint (Jack 1953). Standing lateral radiographs can be used to classify the deformity into one of three anatomical types (Jack 1953). The first is associated with an isolated talonavicular break, and the talus is more vertical than normal because of severe laxity of the ligamentous and tendinous supports of the subtalar and talonavicular joints. The second type has an isolated naviculocuneiform break and the third has a combined break

R. K. Fraser, FCS SA(Orth), MMed, Clinical Orthopaedic Fellow M. B. Menelaus, MD, FRCS, FRACS, Senior Orthopaedic Surgeon P. F. Williams, FRCS, FRACS, Senior Orthopaedic Surgeon Department of Orthopaedics, Royal Children's Hospital, Parkville, Victoria 3052, Australia.

W. G. Cole, MSc, PhD, FRACS, FRCS C, Head and Professor of Surgery

Division of Orthopaedics, The Hospital for Sick Children, 555 University Avenue, Toronto, Ontario. Canada M5G IX8.

Correspondence should be sent to Professor W. G. Cole.

(1)1995 British Editorial Society of Bone and Joint Surgery $0301-620 X / 95 / 3980 \$ 2.00$ involving both the talonavicular and naviculocuneiform joints.

We have reviewed the surgical management of older children and adolescents with persistent symptoms due to mobile flat feet associated with isolated naviculocuneiform breaks. The medial longitudinal arch can be restored by corrective arthrodesis of the naviculocuneiform joints (Hoke 1931). An alternative is the Miller procedure in which corrective arthrodeses of the joints between the navicular and medial cuneiform and between the first metatarsal and the medial cuneiform are combined with distal advancement of an osteoperiosteal flap based on the tibialis posterior tendon (Miller 1927). A modified Hoke-Miller procedure has been described by Duncan and Lovell (1983). There is also the Durham plasty which combines corrective arthrodesis of the naviculomedial cuneiform joint with proximal and plantar advancement of a medial osteoperiosteal flap and distal and plantar advancement of the tibialis posterior tendon (Caldwell 1953; Coleman 1983).

Miller (1927) reported satisfactory results in all 16 feet that were reviewed up to 2.5 years after his procedure, but no long-term results have been reported. We therefore report our long-term results after this operation in 38 feet.

\section{PATIENTS AND METHODS}

We made a retrospective study of 27 patients who had undergone the Miller operation for mobile flat feet at the Royal Children's Hospital, Melbourne from 1965 to 1987. Twenty-two of the patients $(82 \%)$ were reviewed; five could not be traced. Thirteen boys and nine girls had had 38 Miller operations, 16 bilateral and six unilateral. Operation had been at an average age of 13 years ( 10 to 17) and the average follow-up was 12 years ( 3 to 27 ). We chose this minimal period to exceed the longest review reported by Miller (1927).

Indications for surgery. All patients had chronically symptomatic mobile flat feet with convex medial borders. The complaints included foot strain $(87 \%)$, excessive shoe wear $(74 \%)$ and an unsightly appearance $(44 \%)$. In all affected feet, standing lateral radiographs had shown isolated sagging of the medial longitudinal arch at the naviculocuneiform joint (Fig. 1). Surgery was undertaken because orthotic treatment had failed to relieve symptoms. 


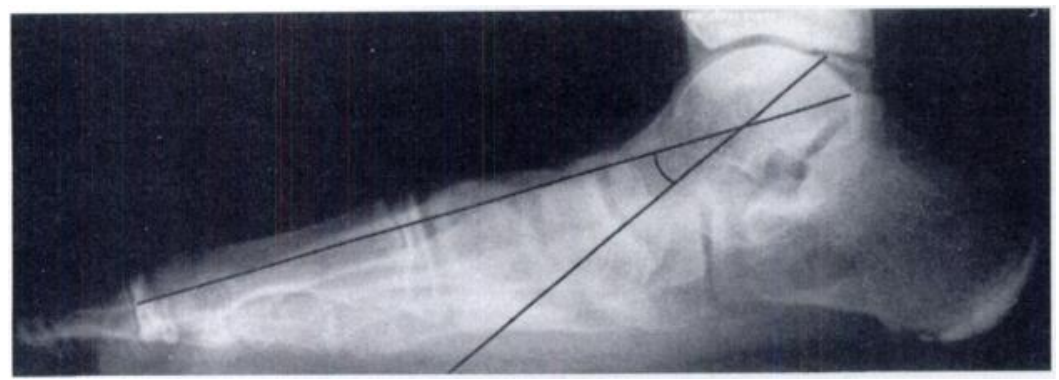

Fig. 1

Figure 1 - Preoperative standing lateral radiograph of a mobile flat foot with a talometatarsal angle of $24^{\circ}$ due to an isolated break at the naviculocuneiform joint. Figure 2 - Postoperative lateral standing radiograph of the same foot showing reconstitution of the medial longitudinal arch.

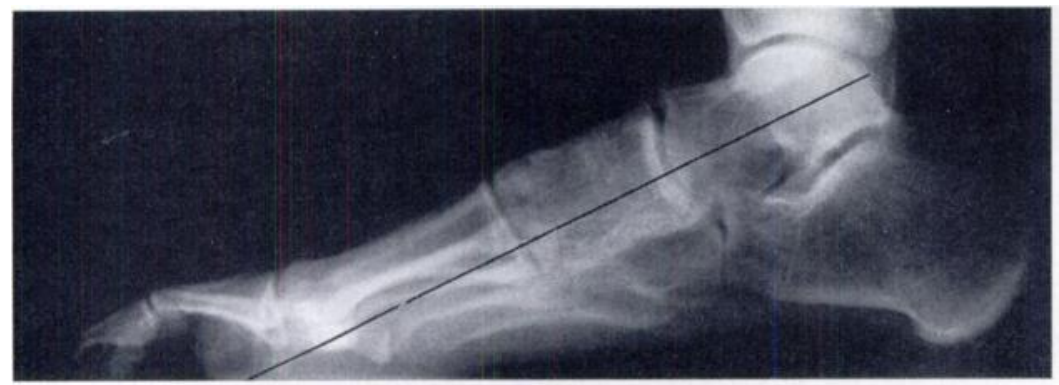

Fig. 2

Operative technique. A curved longitudinal incision is centred over the naviculocuneiform joint and the insertions of tibialis anterior and tibialis posterior are exposed. An osteoperiosteal flap is raised. based proximally on the insertion of tibialis posterior to expose the naviculocuneiform joint. The articular surfaces are excised with the removal of more bone from the plantar than dorsal margins of the joint to restore the medial longitudinal arch by an arthrodesis (Fig. 2). Miller (1927) included also an arthrodesis of the first metatarsocuneiform joint, and this second arthrodesis was done in seven feet $(18 \%)$ of our series.

The exposed bone surfaces are compressed by the distal advancement of the osteoperiosteal flap beneath the tibialis anterior tendon. The tendo Achillis is lengthened if there is any fixed equinus deformity: this was required in 35 feet $(92 \%)$. The wound is closed and a below-knee cast is applied using the Hoke moulded-sole plaster technique (Hoke 1931). This is first applied to the foot with the heel in varus to relax the tibialis posterior; the first metatarsal is then plantar-flexed to restore the medial longitudinal arch. When this part of the cast has set, the ankle is dorsiflexed to a neutral position without elevating the first metatarsal, and the cast is completed. The cast is retained for eight weeks and a medial arch support is worn for another three months.

Clinical assessment. We used a graphic rating method to compare pre- and postoperative disability (Huskisson 1974). We recorded the medial longitudinal arch as grade 1 when it was normal or slightly depressed on weight-bearing, as grade 2 when it was absent but the medial border of the foot was straight, and grade 3 when there was no arch and a convex medial border (Jack 1953). We also recorded the position of the heel, the range of motion of the subtalar, midtarsal and ankle joints and the power of tibialis posterior.

The clinical result was graded excellent if the patient had no pain, disability or excessive shoe wear and was pleased with the appearance of the foot, good if there were mild symptoms after prolonged activity, or poor if there had been no improvement in the patient's symptoms. Excellent and good results were regarded as satisfactory.

Radiological assessment. Anteroposterior and lateral standing radiographs of the feet were taken. The talometatarsal angle was measured on the lateral radiograph at the intersection of the long axis of the talus with the long axis of the first metatarsal (Fig. 1). The angle was positive in pes cavus and negative in pes planus. Angles between $+4^{\circ}$ and $-4^{\circ}$ were accepted as indicating a normal weightbearing medial longitudinal arch (Gould 1983). Any break of the naviculocuneiform and talonavicular joints was also determined from the lateral standing radiographs. A vertical line through the centre of the navicular and parallel to its proximal articular surface is normally perpendicular to the talometatarsal axis on the lateral standing radiograph (Giannestras 1976). If not, there is a break at either the talonavicular, naviculocuneiform or at both joints. Union 
Table I. Clinical and radiological results by number of feet

\begin{tabular}{|c|c|c|}
\hline \multirow[b]{2}{*}{ Variable } & \multicolumn{2}{|c|}{ Clinical result } \\
\hline & $\begin{array}{l}\text { Satisfactory } \\
(\mathrm{n}=32)\end{array}$ & $\begin{array}{l}\text { Poor } \\
(n=6)\end{array}$ \\
\hline \multicolumn{3}{|l|}{ Medial arch* } \\
\hline Grade 1 & 14 & 3 \\
\hline 2 & 15 & 2 \\
\hline 3 & 3 & 1 \\
\hline \multicolumn{3}{|c|}{ Talometatarsal angle } \\
\hline Normal & 10 & 3 \\
\hline Increased & 15 & 2 \\
\hline \multicolumn{3}{|c|}{ Naviculocuneiform joint } \\
\hline United & 17 & 5 \\
\hline Ununited & 8 & 0 \\
\hline
\end{tabular}

* see text for definitions

and nonunion of the naviculocuneiform joint and osteoarthritis of adjoining joints were also recorded (Seymour 1967).

\section{RESULTS}

Clinical results. Twenty-four $(63 \%)$ feet were excellent, eight $(21 \%)$ were good and six $(16 \%)$ were poor at the late review. Poor results had been evident during rehabilitation and were not due to late deterioration of originally satisfactory results.

The medial longitudinal arch was grade 3 in all feet preoperatively and had improved to grades 1 and 2 in 34 of the feet (89\%; Table I). All feet had MRC grade 5 power in tibialis posterior (Medical Research Council 1942). Mild valgus was seen in two heels and midtarsal movement was restricted in one foot. No foot showed any fixed equinus.

Radiological results. At the late review, standing radiographs were taken of 30 feet (79\%), not being performed in four pregnant women ( 8 feet) who had had bilateral Miller operations. There were normal talometatarsal angles in 13 of the feet (43\%; Fig. 2). Seventeen feet had an abnormal talometatarsal angle due to a break at the naviculocuneiform joint, but none had a break in the medial longitudinal arch at the talonavicular or first metatarsocuneiform joints. Some of the preoperative radiographs were missing, and we were unable therefore to determine whether the abnormal talometatarsal angles were due to failure to achieve correction or to subsequent loss of correction.

There was nonunion of the naviculocuneiform joint in eight feet $(21 \%)$. The intermediate cuneiform was included in the fusion in ten feet $(26 \%)$ and the first metatarsal was included in seven feet $(18 \%)$. The additional arthrodeses had united except for one first metatarsocuneiform arthrodesis.

Mild degenerative changes, including joint-space narrowing, sclerosis and osteophytes, were noted in the talonavicular and calcaneocuboid joints of one foot. The foot was graded as having a good clinical result.

Combined results. In total, 32 feet $(84 \%)$ were clinically satisfactory and six (16\%) were poor. Univariate statistical analyses of the grade of medial arch, talometatarsal angle, union of the arthrodesis, extent of the arthrodesis, heel valgus, fixed equinus, joint stiffness and radiological signs of osteoarthritis at late review showed no significant differences when feet with a satisfactory clinical outcome were compared with those with a poor clinical outcome. For this reason, multivariate analyses were not undertaken.

\section{DISCUSSION}

Our study shows that a high proportion of satisfactory clinical results can be achieved for up to 27 years after the Miller operation has been performed during adolescence, and adds to the short-term report by Miller (1927). We have found no other reports of the short or long-term outcomes.

We used the operation infrequently, and only for adolescent patients with persistent symptoms in mobile flat feet who showed a break of the naviculocuneiform joint. The talonavicular joint was normal in all these feet, and is usually abnormal in most patients with severe symptoms from mobile flat feet (Butte 1937). The chronic preoperative complaints of foot strain, excessive shoe wear and unsightly appearance were improved in $84 \%$ of feet, but symptoms persisted in $16 \%$ of the feet for no apparent clinical or radiological reason.

Similar long-term results have been achieved with the Durham plasty. Caldwell (1953) and Coleman (1983) reported satisfactory results in $95 \%$ of feet followed for up to 6 and 26 years, respectively. The Hoke procedure alone is inadequate, since after two to nine years, satisfactory clinical and radiological results were reported in only $67 \%$ of feet (Butte 1937) and by 16 to 19 years, there was osteoarthritis of the talonavicular and subtalar joints in $69 \%$ of feet (Seymour 1967).

It is probable that the high proportion of long-term successful results after the Miller and Durham procedures is due to the tightening of the ligaments along the medial and plantar surfaces of the medial longitudinal arch, and to the advancement of the tibialis posterior tendon. These soft-tissue procedures appear to protect the tarsal joints from osteoarthritis.

In our series, $21 \%$ of the arthrodeses had failed to unite although all such feet had clinically satisfactory results. It seems that inadequate compression of the cancellous bone surfaces was achieved by advancement of the osteoperiosteal flap and moulding of the cast. It has been suggested that internal fixation with a small compression screw, as in the modified Hoke-Miller procedure, would provide better compression and a higher rate of union (Duncan and Lovell 1983).

No benefits in any form have been received or will be received from a commercial party related directly or indirectly to the subject of this article. 


\section{REFERENCES}

Butte FL. Navicular-cuneiform arthrodesis for flat-foot: an end-result study. J Bone Joint Surg 1937:19:496-502.

Caldwell GD. Surgical correction of relaxed flatfoot by the Durham flatfoot plasty. Clin Orthop 1953;2:221-6.

Coleman SS. Complex foot deformities in children. Philadelphia, Lea and Febiger, 1983.

Duncan JW, Lovell WW. Modified Hoke-Miller flatfoot procedure. Clin Orthop 1983:181:24-7.

Gould N. Evaluation of hyperpronation and pes planus in adults. Clin Orthop 1983:181:37-45.

Giannestras NJ. Foot disorders: medical and surgical management. Philadelphia, etc: Lea and Febiger, 1973.
Hoke M. An operation for the correction of extremely relaxed flat feet. $J$ Bone Joint Surg 1931;13:773-83.

Huskisson EC. Measurement of pain. Lancet 1974;ii:1127-31.

Jack EA. Naviculo-cuneiform fusion in the treatment of flat foot. $J$ Bone Joint Surg [Br] 1953;35-B:75-82.

Medical Research Council. Aids to the investigation of peripheral nerve injuries. Med Res Council War Memo No. 71942.

Miller OL. A plastic flat foot operation. $J$ Bone Joint Surg 1927:9:84-91.

Seymour N. The late results of naviculo-cuneiform fusion. $J$ Bone Joint Surg [Br] 1967;49-B:558-9. 